\title{
Enhancing WLAN Capacity by Strategic Placement of Tetherless Relay Points
}

\author{
Aaron So, Student Member, IEEE and Ben Liang, Senior Member, IEEE
}

\begin{abstract}
With the proliferation of wireless local area network (WLAN) technologies, wireless Internet access via public hotspots will become a necessity in the near future. In outdoor areas where the installation of a large number of wired access points is practically or economically infeasible, mobile users located at the edge of the network communicate with the access point at a very low rate and in turn waste network resources. In this work, we promote the use of tetherless relay points (TRPs) to improve the throughput of a WLAN in such environments. We first provide a high level description on how to integrate TRPs in a multi-rate WLAN architecture. We then propose an integer-programming optimization formulation and an iterative approach to compute the best placement of a fixed number of TRPs. Finally, we show in numerical analysis, through a case study based on relay-enabled rate adaptation and IEEE 802.11-like multi-rate physical model with Rayleigh fading, that for a wide range of system parameters, significant performance gain can be achieved when TRPs are strategically installed in the network.
\end{abstract}

Index Terms-Wireless local area network, tetherless relay point, placement optimization, capacity improvement, mathematical programming/optimization.

\section{INTRODUCTION}

Wireless Local Area Networking (WLAN) technologies, such as IEEE 802.11 and HiperLAN/2, provide wireless broadband data access for mobile Internet users and are expected to create a plethora of business opportunities. As a result, products that support WLAN standards and public wireless hotspots have proliferated. Given the explosive growth in wireless hotspot usage, enhancing the capacity of WLANs has become an important issue.

Much research has been carried out on improving the capacity and efficiency of WLANs. In [1], [2], [3], and [4], insights into the efficient operation of large scale hotspot networks were discussed. In [5], [6], and [7], contention-free polling algorithms were proposed for WLANs to ensure high throughput, low latency and energy efficient operation. For the contention mode, carrier sense range adjustment, interference control, and controllable resource allocation methods were proposed in [8], [9], and [10] to improve throughput and system utilization in WLANs.

In environments where wiring is convenient, one obvious solution to improve WLAN capacity is to install more access points (APs) in the network so that the distance between a mobile host (MH) and an access point, which is wired to

Aaron So and Ben Liang are with the Department of Electrical and Computer Engineering, University of Toronto, 10 King's College Road, Toronto, Ontario, M5S 3G4, Canada. E-mail: \{aaronso,liang\}@ comm.utoronto.ca. This work was supported in part by the Natural Sciences and Engineering Research Council of Canada and Bell Canada through its Bell University Laboratories R\&D program. the ISP network, can be reduced. However, in environments where wiring is very costly or impossible, we promote the installation of tetherless relay points (TRPs), which relay data wirelessly between the mobile hosts and the wired access point, to improve WLAN capacity. Such networks require more careful planning in order to utilize the wireless media efficiently. In this work, we consider a TRP as an immobile device which has a high capacity battery or access to the power supply but does not have direct access to the Internet. A TRP helps deliver a MAC-layer data packet from the AP to a distanced MH by first receiving the packet wirelessly from the AP, and then retransmitting it to the $\mathrm{MH}$, or vice versa. Since wireless signal attenuates severely as it travels through a distance, by using a TRP, the resulting bit rate can be much higher than the original one hop bit rate.

In a wireless communication system, due to the strong correlation between distance and data rate, different placements of TRPs can significantly alter the network performance. Much prior research has been carried out on improving the capacity and/or ensuring the efficient operation of relay-enable wireless networks [11][12][13][14][15][16][17]. However, they are not concerned with the question of how to place TRPs in a WLAN such that the throughput capacity is maximized. The main focus of this work is to determine the optimal placement of a given number of TRPs in a WLAN. We propose an analysis and optimization framework that exploits the multi-rate capability of the WLAN physical layer. Our main contributions include:

- New TRP relaying architecture with throughput capacity optimization objectives in a multi-rate WLAN;

- Optimization formulation to derive the optimal placement of the TRPs in a WLAN with or without location restriction;

- Solution to the resulting integer-programming problem through Lagrangian relaxation and a subgradient iterative algorithm adapted to the characteristics of a TRP-based WLAN;

- Simplification methods based on the intrinsic properties of special network environments, which can significantly reduce the computation time of the above iterative algorithm; and

- Numerical investigation into the throughput capacity of a WLAN with TRPs in an IEEE 802.11-like multi-rate physical model with Rayleigh fading, providing design guidelines on how the optimal placement of TRPs is affected by the number of TRPs, transmission power, path loss exponent, and traffic pattern. 
The rest of this paper is organized as follows. In Section II, we review the related work in multihop wireless networks and WLAN research. In Section III, we give a high level description of the relaying architecture and derive our design objective. In Section IV, we cast the general TRP placement optimization problem and show how this problem can be solved by a Lagrangian relaxation iterative algorithm. In Section V, we discuss simplifications based on the intrinsic properties of some common network environments to improve the efficiency of the Lagrangian relaxation iterative algorithm. In Section VI, we present a sample relaying and rate adaptation algorithm and a physical layer rate model for IEEE $802.11 \mathrm{~g}$ WLAN under Rayleigh fading. In Section VII, we discuss the convergence time of the proposed optimization algorithm and show the effects of different system parameters on the strategic placement of the TRPs. Finally, concluding remarks are given in Section VIII.

\section{RELATED WORK}

Motivated by recent advances in ad hoc networking [11][12], there has been much research into using peer mobile hosts to relay data in the context of wireless networks. In [13], the concept of multihop cellular network was introduced. The results of this work showed that using peer mobile hosts to relay data to the base station can improve network performance when compared with the traditional single hop cellular network. In [18], a similar idea with emphasis on coverage extension for WLANs was investigated. In the context of multi-rate WLANs, [14] and [15] showed that by using other mobile hosts to perform relaying, the performance of the network can be improved under the Point Coordination Function (PCF) and the Distributed Coordination Function (DCF), respectively. These prior research efforts motivated our investigation into relay infrastructure in WLANs.

The concept of using immobile nodes to relay traffic has received relatively less attention in the literature. Using immobile relays such as TRPs have several advantages when compared with mobile relay nodes. First, since TRPs are relatively sedentary ${ }^{1}$, it is reasonable to assume that they have access to external power supply or have a large built-in battery. Consequently, unlike in other works that use mobile hosts as relay nodes, energy is not a constraint for TRPs. Second, since the distance between an AP and a TRP is relatively static, links between the AP and the TRPs are more stable. Furthermore, the sedentary TRPs can be configured to their optimal setting which maximizes their benefits.

The benefits of using immobile relay node have been discussed in several contexts. In [16], the $i C A R$ architecture for cellular network was introduced. $i C A R$ uses immobile relay nodes to alleviate traffic congestion by relaying traffic from a congested cell to the less congested neighbor cells. The benefit of using immobile relay nodes is also explored in mesh network research. In [17], innovative integration techniques were developed to minimize the number of access points in a mesh network to reduce wiring cost, while maintaining QoS constraints from all nodes. In [19], a new scalable and

\footnotetext{
${ }^{1}$ They may be moved to follow large time-scale network traffic variations.
}

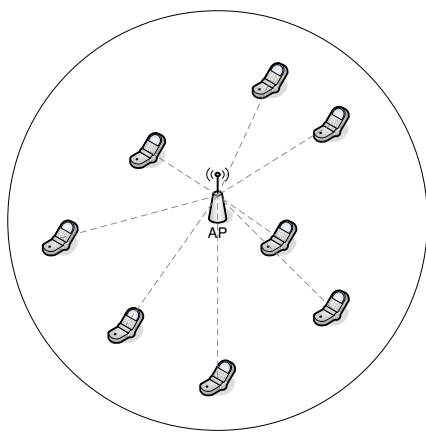

Basic Service Set

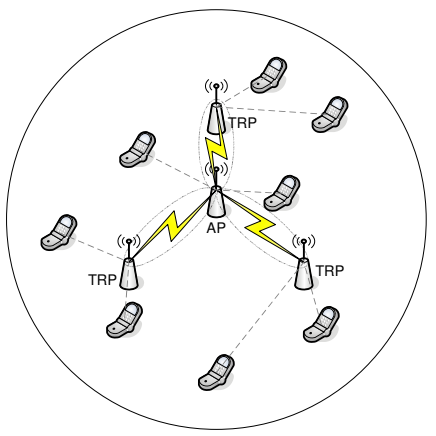

Basic Service Set with TRPs
Fig. 1. Original BSS and BSS with TRPs.

cost effective architecture for wireless mesh network was introduced. To gain insight into the physical environments and deployment challenges, a measurement driven deployment of wireless mesh network was studied in [20]. The issues of routing in mesh network were studied in [21] and [22], where the relationship among throughput and several routing metrics were investigated theoretically and empirically. These research efforts have revealed the benefit of immobile relay nodes in large scale wireless networks. In our research, we explore the potential of immobile relay infrastructure in a single cell.

In [23], we studied the effects of relaying with commercial Extension Points (EPs) and the optimal relay placement in a one-dimensional WLAN, using a divide-and-conquer searching algorithm. In this paper, we study realistic relaying that are similar to the IEEE 802.11 standard, while taking into consideration finite data rates and multi-path fading. We propose a novel iterative integer-programming solution framework to optimize the TRP placement and investigate WLAN performance in a more general two-dimensional setting with or without environmental constraints.

\section{Relaying Architecture And Design ObJectives}

We aim to improve the throughput capacity of a Basic Service Set (BSS) [24] by using TRPs. In a BSS, there is an AP connected with the wired ISP network, and this AP provides wireless coverage to a local area. Moreover, all MHs and the AP are fully connected (i.e., forming a complete graph) via a single channel. Thus, only one transmitter is allowed to transmit at any given time, and simultaneous transmission inside a BSS will result in collision.

We investigate relaying with TRPs in an environment where wiring is practically or economically infeasible, to improve the throughput capacity of a BSS without using additional wireless resources. The AP, TRPs and MHs have to share the same wireless medium originally assigned to the BSS. Fig. 1 shows the difference between the original BSS and a BSS with TRPs.

Since much research has been carried out on implementing relaying mechanisms [14] [15], in the following subsection, we provide only a high level description on how the system should operate. We then derive the objective function that we will minimize regarding the placement of the TRPs. We emphasize that our analytical and optimization framework is valid as long as the relaying mechanism follows the operation principles 
described in this section. An example of such implementation will be presented in Section VI.

\section{A. Operational Procedure Overview}

We consider the single BSS scenario, which is sufficient to provide design guidelines even in cases where multiple BSSs co-exist. First, if the BSSs are sparse, then the interference between them can be negligible. Second, for densely installed BSSs, a practical implementation is likely to allocate noninterfering channels between neighboring BSSs. Third, if two interfering BSSs must be installed as neighbors, then two cases should be considered. If the neighboring BSSs use the same channel, then the channel must be time-shared. In this case, a TRP would be active only at the same time that its associated AP is active, and the optimal TRP configuration would be the same as if the BSS were in isolation. If the neighboring BSSs use different but interfering channels, the data rate and hence the packet transaction time would be affected by such interference. In this case, the expected packet transaction time should account for the lower bit rate due to interference.

To utilize the TRPs intelligently, MHs located at different locations should select the most appropriate TRP to forward packets. $^{2}$ If a TRP is selected to assist a MH, downlink packets from the wired network will be delivered from the AP to this TRP, and this TRP will deliver the packet to the MH. The reverse is performed for uplink packets. In order to take full advantage of the TRP, a central entity should have up-to-date link statistics and be able to make the selection decisions for the MHs. For example, such information can be compiled in a report and send to the AP periodically. By collecting the link quality information, the AP can decide which TRP (or the AP itself) each $\mathrm{MH}$ should select to maximize its throughput. The AP could inform the MH about its selection decision via a special control message or could embed such information in existing control packets such as RTS, CTS, ACK or POLL. We emphasize here that the exact implementation details can be flexible because the main focus of this work is on the optimal placement of TRPs. In our analytical framework, we only assume that a link quality estimation procedure exists and the AP and MHs are able to decide which TRP to use in order to maximize their throughput. ${ }^{3}$

Once a TRP is assigned to each $\mathrm{MH}$, a rate adaptation mechanism such as [25][26] should be used by the MH, TRP, and AP to decide the communication rate of each link. Again, the exact details of the rate adaptation scheme can be flexible. Our analytical framework only assumes that a rate adaptation mechanism is used, and given the locations of a $\mathrm{MH}$ and a TRP, the average data packet delivery speed between the AP and MH via this TRP can be quantified. We do not expect the operation mechanism of the network to have significant influence on the TRP placement decision because in a fully

\footnotetext{
${ }^{2}$ If none of the TRPs are useful for a particular MH, this MH will not use any TRP.

${ }^{3}$ The above information collection and TRP selection procedure can be implemented in the Network Interface Card (NIC) software as an option for operation in a BSS with TRPs. If a MH does not have this functionality, it will associate itself with the AP and behave as a MH in a regular BSS. Thus, the system is backward compatible.
}

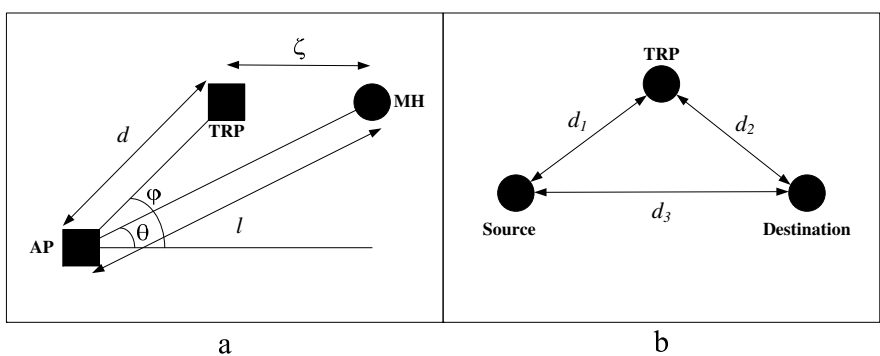

Fig. 2. Single user scenario with one TRP.

connected network, packet collision and other inefficiencies due to polling or distributed contention are mainly affected by the adopted protocols and the number of nodes in the network, but not the location of these nodes. In Section VI, a sample link information collection and relay-enable rate adaptation scheme will be described.

\section{B. Optimization Objective}

TRPs should be placed in locations resulting in the smallest average raw packet transmission time between the AP and different MHs. Before we express this optimization objective mathematically, let us consider the following system.

As shown in Fig. 2a, for the simplest scenario, there is an access point located at the origin, a single $\mathrm{MH}$ located at $(l, \theta)$, and a TRP located at $(d, \varphi) .{ }^{4}$ We denote the lengths of the uplink and downlink packets by $x_{u}$ and $x_{d}$ respectively. The sizes of these packets correspond to the proportion of uplink and downlink traffic. The time axis is divided into time-varying packet transaction cycles. At each cycle, the AP transmits a downlink packet to the MH via the TRP, and then the MH transmits an uplink packet to the AP also via the TRP. Let $t\left(d_{1}, d_{2}, P_{1}, P_{2}, x\right)$ be a random variable representing the transmission time of an $x$-bit packet from a source with reference power $P_{1}$ to a destination via a TRP with reference power $P_{2}$. The distance between the source and the TRP and the distance between the TRP and the destination are $d_{1}$ and $d_{2}$ respectively. Fig. $2 \mathrm{~b}$ describes the physical configuration of the above setting. Let us denote the reference power of the AP, TRP and MH as $P_{a}, P_{t}$ and $P_{m}$ respectively. Let $t_{t r p}^{i}(l, d, \theta, \varphi)$ be a random variable representing the time for a MH located at $(l, \theta)$ to complete the $i^{\text {th }}$ packet transaction cycle via the TRP at $(d, \varphi)$. Then, we have

$$
t_{t r p}^{i}(l, d, \theta, \varphi)=t\left(d, \zeta, P_{a}, P_{t}, x_{d}\right)+t\left(\zeta, d, P_{m}, P_{t}, x_{u}\right)
$$

where $\zeta=\sqrt{l^{2}+d^{2}-2 l d \cos |\theta-\varphi|}$. Then, by the Law of Large Numbers [27], the throughput capacity of the MH with this particular TRP placement, $(d, \varphi)$, is

$$
C(d, \varphi)=\lim _{n \rightarrow \infty} \frac{n x}{\sum_{i=1}^{n} t_{t r p}^{i}(l, d, \theta, \varphi)}=\frac{x}{E\left[t_{t r p}^{i}(l, d, \theta, \varphi)\right]},
$$

where $x=x_{d}+x_{u}$. Therefore, in order to maximize the throughput capacity of the network, we need to find the optimal $(d, \varphi)$ such that $E\left[t_{t r p}^{i}(l, d, \theta, \varphi)\right]$, which we call the expected packet transaction time in this paper, is minimized.

\footnotetext{
${ }^{4}$ Locations are expressed in polar coordinates in this paper.
} 
Hence, our optimization objective of this simple case is to minimize

$$
\begin{aligned}
T_{t r p}(l, d, \theta, \varphi) & =E\left[t_{t r p}^{i}(l, d, \theta, \varphi)\right] \\
& =E\left[t\left(d, \zeta, P_{a}, P_{t}, x_{d}\right)+t\left(\zeta, d, P_{m}, P_{t}, x_{u}\right)\right] \\
& =T\left(d, \zeta, P_{a}, P_{t}, x_{d}\right)+T\left(\zeta, d, P_{m}, P_{t}, x_{u}\right),
\end{aligned}
$$

where $T\left(d_{1}, d_{2}, P_{1}, P_{2}, x\right)$ is the expected value of $t\left(d_{1}, d_{2}, P_{1}, P_{2}, x\right)$, which we call the expected packet transmission time.

For any particular TRP placement, MHs will choose the most appropriate TRP to use or communicate directly with the AP. Hence, MHs at different locations have different expected packet transaction times. Since in a typical WLAN environment, the location of the MHs can be described only probabilistically, for a WLAN with multiple MHs, our minimization objective is the average expected packet transaction time in the network, which we called network expected packet transaction time. Note that this objective can accommodate any weighted fairness model among the mobile users, by assigning more packet transmission opportunity to some MHs. In the rest of this paper, for illustration simplicity, we assume strict fairness among all users, which can be achieved by a centralized round-robin scheme or a distributed fair queuing mechanism.

In Section IV, we propose an optimization framework for the placement of TRPs in a multi-user WLAN environment for a generic function $T\left(d_{1}, d_{2}, P_{1}, P_{2}, x\right)$, which can be obtained from theoretical models or by regression models based on sitesurvey results, accounting for noise and possible interferences. Then, in Section VI, we further derive $T\left(d_{1}, d_{2}, P_{1}, P_{2}, x\right)$ based on a sample relaying and rate adaptation mechanism and an IEEE $802.11 \mathrm{~g}$ physical model with large-scale propagation path loss and Rayleigh fading.

\section{TRP PLACEMENT OPTIMIZATION}

In this section, we first provide an analytical framework to analyze the network expected packet transaction time with respect to different TRP placements. This analytical framework is then used to cast the TRP placement problem as an optimization problem. Finally, we present an efficient solution based on Lagrangian relaxation and a subgradient algorithm.

\section{A. Throughput Capacity Maximization with Multiple TRPs}

We assume that a fixed number, $N$, where $N>2$, of TRPs are available for each AP in the WLAN system. Fig. 3 illustrates a simple example, where an AP is installed at the center of a park, and this AP provides wireless coverage within a cell of radius $L$. To enhance the throughput capacity of the network, TRPs are installed around this AP. A vector, $\underline{d}$, is used to represent the displacement of TRPs with respect to the AP. Moreover, a vector $\underline{\varphi}$ is used to represent the angle between a predefined reference base line and the radial lines

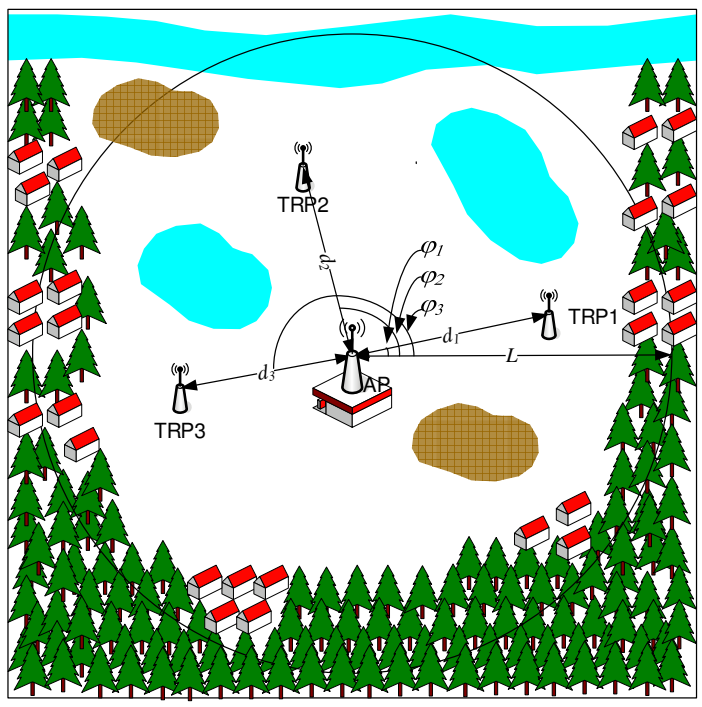

Fig. 3. Multi-user two-dimensional WLAN with multiple TRPs.

where the TRP reside. Thus, we have

$$
\underline{d}=\left(\begin{array}{c}
d_{1} \\
d_{2} \\
\vdots \\
d_{N}
\end{array}\right), \quad \underline{\varphi}=\left(\begin{array}{c}
\varphi_{1} \\
\varphi_{2} \\
\vdots \\
\varphi_{N}
\end{array}\right),
$$

where $0<d_{i} \leq L, \forall i$, and $0 \leq \varphi_{1} \leq \varphi_{2} \leq \cdots \leq \varphi_{N} \leq 2 \pi$.

The MHs are distributed in the coverage area of the network with the probability density function $f(l, \theta)$. This density function may not be uniform. For example, the users will not be located in the ponds or the river, and are more likely to be located around special attractions. A MH may either communicate with the AP directly or communicate with the AP via the TRP whichever resulting in the lowest expected packet transaction time. Therefore, the network expected packet transaction time for a particular TRP placement can be computed as

$$
\begin{aligned}
& \overline{T_{\operatorname{trp}(\underline{d}, \underline{\varphi})}}= \\
& \int_{0}^{2 \pi} \int_{0+\epsilon}^{L} f(l, \theta) \min \left[T_{a p}(l), \min _{1 \leq k \leq N} T_{t r p}\left(l, d_{k}, \theta, \varphi_{k}\right)\right] d l d \theta,
\end{aligned}
$$

where $\epsilon>0$ is small, and $T_{a p}(l)$ represents the expected packet transaction time when the MH is directly communicating with the AP. Similar to the expected packet transaction time derived in the previous section for $T_{t r p}(l, d, \theta, \varphi)$, we have

$$
T_{a p}(l)=T\left(l, P_{a}, x_{d}\right)+T\left(l, P_{m}, x_{u}\right),
$$

where $T(l, P, x)$ is the expected transmission time of an $x$-bit packet from a transmitter with reference power $P$ to a receiver $l$ meters away.

In most network environments, there are some areas where the installation of a TRP is prohibited. For example, in Fig. 3, it is not possible to install a TRP in the ponds. Let us defined the feasible areas for the installation of a TRP be $S$. Using 


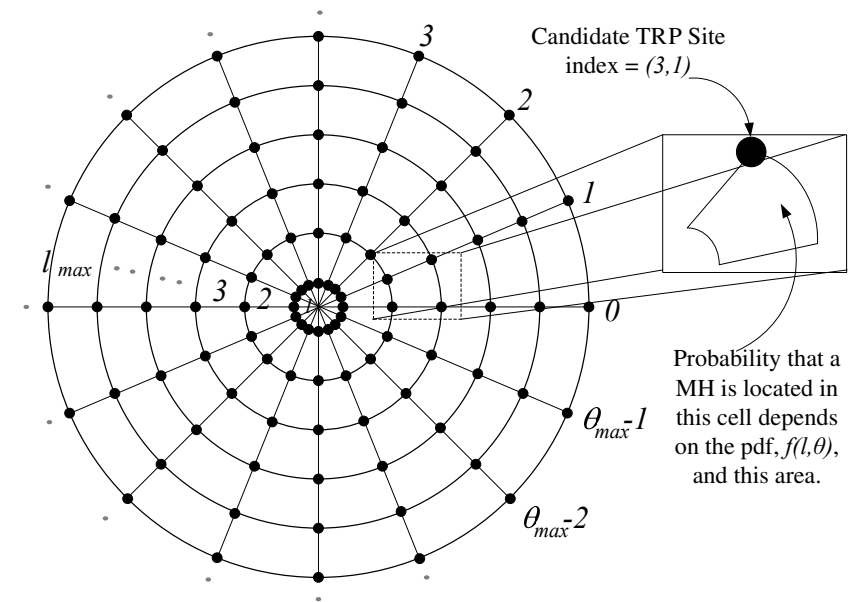

Fig. 4. Discretization of the network.

(5), we have the following optimization problem:

$$
\begin{aligned}
\text { Objective: } & \min _{\underline{d}, \underline{\varphi}} \overline{T_{\operatorname{trp}(\underline{d}, \underline{\varphi})}} \\
\text { s.t. } & 0<d_{i} \leq L, \quad \forall i \\
& 0 \leq \varphi_{1} \leq \varphi_{2} \leq \cdots \leq \varphi_{N} \leq 2 \pi \\
& \left(d_{i}, \varphi_{i}\right) \in S, \quad \forall i .
\end{aligned}
$$

Clearly, this problem is difficult to solve directly. In reality, there is no need to determine the TRP placement with infinite granularity. Hence, we can calculate the approximate value of (5) by discretizing the network into a large but finite number of areas, where a mobile host is located at each area with a certain probability. Then, the integral in (5) can be interpreted as a Riemann sum. Next, we reformulate (7) into a discrete form similar to the $p$-median problem with an additional constraint.

\section{B. Problem Reformulation}

As shown in Fig. 4, we can divide the entire network into $\theta_{\max }$ equally sized sectors, and each sector is then divided into $l_{\max }$ equal length cells. A MH is located in each cell with a certain probability, and if a MH occupies a cell, we assume that it is located at the outer-left corner. Moreover, the outer-left corner of each cell also represents a candidate site of the TRP set. Thus, each MH or TRP candidate site can be uniquely identified by its radial line number and its cell number. For example, the selected site in Fig. 4 lies on the third cell of the first radial line, so this site is indexed by $(3,1)$. For notation purpose, we use $(i, j)$ to describe the location of a $\mathrm{MH}$, while we use $(\delta, \tau)$ to represent the location of a TRP candidate site.

We define the following notations:

$$
\begin{aligned}
\Delta \theta & =\frac{2 \pi}{\theta_{\max }}, \Delta l=\frac{L}{l_{\max }}, \\
d_{k} & \approx \delta_{k} \Delta l, \quad 1 \leq k \leq N, \quad \delta_{k} \in \mathbf{Z}^{+}, \quad 1 \leq \delta_{k} \leq l_{\max }, \\
\varphi_{k} & \approx \tau_{k} \Delta \theta, \quad 1 \leq k \leq N, \tau_{k} \in \mathbf{Z}, \quad 0 \leq \tau_{k} \leq \theta_{\max }-1, \\
\underline{\delta} & =\left(\delta_{1}, \ldots, \delta_{N}\right)^{T}, \quad \underline{\tau}=\left(\tau_{1}, \ldots, \tau_{N}\right)^{T},
\end{aligned}
$$

$$
\begin{gathered}
h_{(a, b)}=\int_{b \Delta \theta}^{(b+1) \Delta \theta} \int_{(a-1) \Delta l}^{a \Delta l} f(l, \theta) d l d \theta, \\
a=1, . ., l_{\max }, \text { and } b=0, . ., \theta_{\max }-1, \\
S^{\prime}=M(S),
\end{gathered}
$$

where $M(\cdot)$ is the mapping of a set from the continuous space to the discrete space. Furthermore, for notation simplification, we denote

$$
\begin{aligned}
T_{a p}^{\Delta l}(i) & =T_{a p}(i \Delta l), \\
T_{t r p}^{\Delta l, \Delta \theta}(i, \delta, j, \tau) & =T_{t r p}(i \Delta l, \delta \Delta l, j \Delta \theta, \tau \Delta \theta) .
\end{aligned}
$$

Then, (5) can be approximated as

$$
\begin{aligned}
& \overline{T_{t r p}(\underline{\delta}, \underline{\tau})} \\
& \approx \sum_{i=1}^{l_{\max }} \sum_{j=0}^{\theta_{\max }-1} \min \left[T_{a p}^{\Delta l}(i), \min _{1 \leq k \leq N} T_{t r p}^{\Delta l, \Delta \theta}\left(i, \delta_{k}, j, \tau_{k}\right)\right] h_{(i, j)}
\end{aligned}
$$

To facilitate the minimization of $\overline{T_{t r p}(\underline{\delta}, \underline{\tau})}$, we define two sets of decision variables, $\mathbf{X}$ and $\mathbf{Y}$, such that

$$
X_{(\delta, \tau)}=\left\{\begin{array}{ll}
1 & \text { TRP is placed in position }(\delta, \tau) \\
0 & \text { otherwise }
\end{array},\right.
$$

$Y_{(i, j),(\delta, \tau)}= \begin{cases}1 & \mathrm{MH}(i, j) \text { is served by TRP }(\delta, \tau) \\ 0 & \text { otherwise. }\end{cases}$

Note that $X_{(0,0)}=1$ because the access point is always present. Moreover, since $X_{(0,0)}=1$, we have $Y_{(i, j),(0,0)}=1$ only if the MH at $(i, j)$ is served directly by the AP.

Therefore, (7) can be reformulated as

$$
\begin{aligned}
\min _{\mathbf{X}, \mathbf{Y}}: \quad & \sum_{i=1}^{l_{\max }} \sum_{j=0}^{\theta_{\max }-1}\left[h_{(i, j)} T_{a p}^{\Delta l}(i) Y_{(i, j),(0,0)}+\right. \\
& \left.\sum_{\delta=1}^{l_{\max }} \sum_{\tau=0}^{\theta_{\max }-1} h_{(i, j)} T_{t r p}^{\Delta l, \Delta \theta}(i, \delta, j, \tau) Y_{(i, j),(\delta, \tau)}\right](11) \\
\text { s.t. } \quad & Y_{(i, j),(0,0)}+\sum_{\delta=1}^{l_{\max }} \sum_{\tau=0}^{\max -1} Y_{(i, j),(\delta, \tau)}=1 \\
& \sum_{\delta=1}^{l_{\max }} \sum_{\tau=0}^{\theta_{\max }-1} X_{(\delta, \tau)}=N \quad \forall \quad(i, j) \\
& X_{(0,0)}=1 \\
& Y_{(i, j),(\delta, \tau)}-X_{(\delta, \tau)} \leq 0 \quad \forall \quad(i, j),(\delta, \tau)(15) \\
& X_{(\delta, \tau)}=0 \quad \forall \quad(\delta, \tau) \notin S^{\prime} \quad(16)
\end{aligned}
$$

Objective (11) minimizes the sum of the approximated version of the network expected packet transaction time. Constraint (12) requires each $\mathrm{MH}$ to be assigned to exactly one TRP or the AP. Constraint (13) states that exactly $N$ TRPs are to be placed. Constraint (14) states that the AP is always present. Constraint $(15)$ requires that the $\mathrm{MH}$ at $(i, j)$ can be assigned to a TRP at $(\delta, \tau)$ only if a TRP is installed at location $(\delta, \tau)$. Finally, constraint (16) required that the TRPs are not located in the infeasible sites. Note that the above 
formulation is similar to the discrete $p$-median problem [28], with an additional constraint (14).

Because of the binary constraints (9) and (10), the formulation above cannot be solved with standard linear programming techniques. Next, we present a Lagrangian relaxation iterative algorithm that allows us to approach the optimal solution from both above and below.

\section{An Optimization-Based Lagrangian Relaxation Iterative Algorithm}

Lagrangian relaxation with subgradient iteration is a technique that can be used to provide approximate solutions to many NP-hard problems efficiently. Thus, noting the distinctive characteristics of our TRP placement formulation, we propose to solve the optimization problem in (11)-(16) by an iterative algorithm as follows.

1) Setting up: We relax constraint (12) and obtain (17), where $\lambda_{(i, j)}$ are the Lagrange multipliers. ${ }^{5}$

2) Solving the simplified problem: For fixed values of the Lagrange multipliers, we wish to minimize the objective function (17). Since the values of $\lambda_{(i, j)}$ are fixed, the first term in the objective function, which is just the sum of all Lagrangian multipliers, is a constant. To minimize (17), we begin by computing the value of

$$
V_{(\delta, \tau)}=\sum_{i=1}^{l_{\max }} \sum_{j=0}^{\theta_{\max }-1} \min \left(0,\left[h_{(i, j)} T_{t r p}^{\Delta l, \Delta \theta}(i, \delta, j, \tau)-\lambda_{(i, j)}\right]\right)
$$

for each candidate TRP location $(\delta, \tau) \in S^{\prime}$. We then find the $N$ smallest values of $V_{(\delta, \tau)}$ and set the corresponding values of $X_{(\delta, \tau)}=1$ and all other values of $X_{(\delta, \tau)}=0$. We then set

$Y_{(i, j)(\delta, \tau)}= \begin{cases}1 & \begin{array}{l}\text { if } h_{(i, j)} T_{t r p}^{\Delta l, \Delta \theta}(i, \delta, j, \tau)-\lambda_{(i, j)}<0 \\ \text { and } X_{(\delta, \tau)}=1\end{array} \\ 0 & \text { otherwise. }\end{cases}$

Moreover, since $X_{(0,0)}=1$, we set

$$
Y_{(i, j)(0,0)}= \begin{cases}1 & \text { if } h_{(i, j)} T_{a p}^{\Delta l}(i)-\lambda_{(i, j)}<0 \\ 0 & \text { otherwise. }\end{cases}
$$

3) Updating the lower and upper bounds: For each iteration of this process, an upper bound and a lower bound of the original objective function (11) need to be determined. The upper bound is a sub-optimal solution that has been discovered which meets the constraints of the original unmodified problem from Step 2, where the AP and $N$ TRP candidate sites are selected. This upper bound to the network expected packet transaction time can be calculated by using (8). Furthermore, as shown in [29], a lower bound for the current iteration is simply the objective function (17) with the values of $\mathbf{X}$ and $\mathbf{Y}$ found in Step 2.

4) Modifying the Lagrange multipliers: The Lagrange multipliers are revised using a standard subgradient optimization procedure. At the $n^{t h}$ iteration of the Lagrangian procedure, we first compute the step size by (19), where $U B$ and $\mathcal{L}^{n}$ are the best upper and lower bounds found so far

${ }^{5}$ In our numerical analysis, all $\lambda_{(i, j)}$ values are initialized to 5000 . up to the current iteration, $Y_{(i, j),(\delta, \tau)}^{n}$ is the optimal value of $Y_{(i, j),(\delta, \tau)}$ at the $n^{t h}$ iteration, and $A^{n}$ is a constant updated as follows. We begin with $A^{1} \leq 2$ an arbitrary small positive number. At each iteration, the value of $A^{n}$ is halved if $\mathcal{L}^{n}$ has not increased in $c_{A}$ consecutive iterations. ${ }^{6}$ Then, the Lagrangian multipliers are updated by (20).

5) Iteration and termination: The algorithm terminates when any one of the following conditions is true:

- A predefined number of iterations are completed.

- The upper bound equals or is close enough to the lower bound.

- $A^{n}$ is small, such that the changes in $\lambda_{(\delta, \tau)}$ becomes too small. Such small changes are not likely to help solve the problem.

Otherwise, we repeat from Step 2.

As shown in Section VII, for all system parameters tested, the difference between the upper bound and the lower bound always converges to less than $2 \%$ within 200 iterations.

\section{COMPLEXITy REDUCTION UNDER UNIFORMITY CONDITIONS}

In some common network environments, the computation complexity of the Lagrangian relaxation iterative algorithm in Section IV can be further reduced. We notice that in many outdoor open environments, we may assume that the mobile hosts are uniformly distributed in the network coverage area, i.e. ${ }^{7}, f(l, \theta)=\frac{l}{\pi L^{2}}$. Moreover, such environments usually do not have much restriction on the TRP candidate sites. Under these conditions, we present the following simplifications technique to reduce the runtime of the proposed algorithm.

We note that in the Lagrangian relaxation iterative algorithm, the most computationally heavy part is in Step 2 of Section IV-C, which ranks all the TRP candidate sites. The purpose of this step is to obtain the subgradient of (17) at $\lambda$. To decrease the computational time, we first reduce the computational effort of computing each $V(\delta, \tau)$. Then, we develop an adaptive sector-based algorithm to minimize the number of TRP candidate sites that we need to rank while finding the subgradient correctly.

\section{A. Reducing the Computational Effort for $V(\delta, \tau)$}

As illustrated in Fig. 5, intuitively, given a TRP placed anywhere on a radial line, say $\tau$, there exists a region defined by $\xi$, where the TRP will not provide any benefit for any MHs located outside this region. The following theorem formalizes this.

Theorem 1: Given a TRP placed on radial line $\tau$, there exists a minimum $\xi$, such that $\forall j \in\left(\xi, \theta_{\max }-\xi\right)$, $T_{t r p}^{\Delta l, \Delta \theta}(i, \delta, j \oplus \tau, \tau)>T_{a p}^{\Delta l}(i)$, for all $i$ and $\delta$, where $a \oplus b=$ $(a+b) \bmod \theta_{\max }$.

The proof of this theorem is given in Appendix I. By using Theorem 1, the effective coverage of a TRP is limited. Therefore, we can set $T_{t r p}^{\Delta l, \Delta \theta}(i, \delta, j, \tau)=\infty$, if $\min (\mid j-$

\footnotetext{
${ }^{6}$ In our numerical analysis, we use $A^{1}=2$ and $c_{A}=4$.

${ }^{7}$ When $f(l, \theta)=\frac{l}{\pi L^{2}}, h_{(a, b)}$ does not depend on $b$. Therefore $h_{(a, b)}=$ $h_{a}$ for all $a \in\left[1, l_{\max }\right]$ and $b \in\left[0, \theta_{\max }\right)$.
} 


$$
\begin{aligned}
\max _{\lambda} \min _{\mathbf{X}, \mathbf{Y}} & \sum_{i=1}^{l_{\max }} \sum_{j=0}^{\theta_{\max }-1} \lambda_{(i, j)}\left[1-Y_{(i, j),(0,0)}-\sum_{\delta=1}^{l_{\max }} \sum_{\tau=0}^{\theta_{\max }-1} Y_{(i, j),(\delta, \tau)}\right]+ \\
& \sum_{i=1}^{l_{\max }} \sum_{j=0}^{\theta_{\max }-1}\left[h_{(i, j)} T_{a p}^{\Delta l}(i) Y_{(i, j),(0,0)}+\sum_{\delta=1}^{l_{\max }} \sum_{\tau=0}^{\theta_{\max }-1} h_{(i, j)} T_{t r p}^{\Delta l, \Delta \theta}(i, \delta, j, \tau) Y_{(i, j),(\delta, \tau)}\right] \\
= & \sum_{i=1}^{l_{\max }} \sum_{j=0}^{\theta_{\max }-1} \lambda_{(i, j)}+\sum_{i=1}^{l_{\max }} \sum_{j=0}^{\theta_{\max -1}}\left[\left(h_{(i, j)} T_{a p}^{\Delta l}(i)-\lambda_{(i, j)}\right) Y_{(i, j),(0,0)}+\right. \\
& \left.\sum_{\delta=1}^{l_{\max }} \sum_{\tau=0}^{\theta_{\max }-1}\left(h_{(i, j)} T_{t r p}^{\Delta l, \Delta \theta}(i, \delta, j, \tau)-\lambda_{(i, j)}\right) Y_{(i, j),(\delta, \tau)}\right]
\end{aligned}
$$

s.t. $\quad(13), \quad(14), \quad(15), \quad(16)$ are satisfied .

$$
t^{n}=\frac{A^{n}\left(U B-\mathcal{L}^{n}\right)}{\sum_{i=1}^{l_{\max }} \sum_{j=0}^{\theta_{\max }-1}\left[Y_{(i, j),(0,0)}^{n}+\sum_{\delta=1}^{l_{\max }} \sum_{\tau=0}^{\theta_{\max }-1} Y_{(i, j),(\delta, \tau)}^{n}-1\right]^{2}}
$$

$$
\lambda_{(i, j)}^{n+1}=\max \left[0, \lambda_{(i, j)}^{n}-t^{n}\left(Y_{(i, j),(0,0)}^{n}+\sum_{\delta=1}^{l_{\max }} \sum_{\tau=0}^{\theta_{\max }-1} Y_{(i, j),(\delta, \tau)}^{n}-1\right)\right]
$$

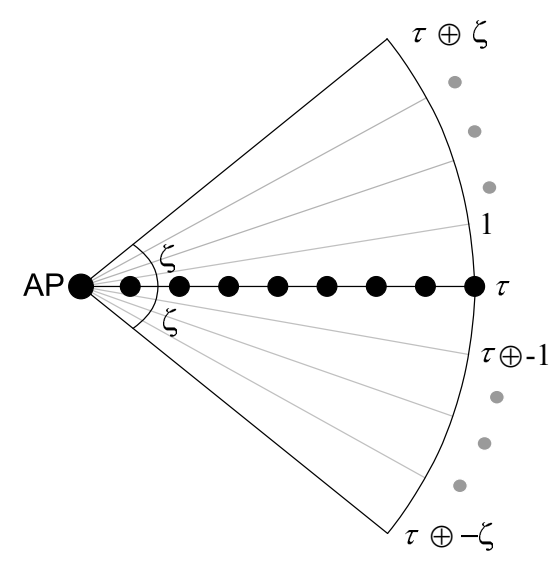

Fig. 5. Effective coverage of a TRP.

$\left.\tau\left|, \theta_{\max }-\right| j-\tau \mid\right)>\xi$, and the resulting output by the algorithm will remain unchanged. Thus, the computation of $V(\delta, \tau)$ can be simplified to

$$
\begin{aligned}
V_{(\delta, \tau)} & =\sum_{i=1}^{l_{\max }} \sum_{j=0}^{\theta_{\max }-1} \min \left(0,\left[h_{i} T_{t r p}^{\Delta l, \Delta \theta}(i, \delta, j, \tau)-\lambda_{(i, j)}\right]\right) \\
& =\sum_{i=1}^{l_{\max }} \sum_{j=-\xi}^{\xi} \min \left(0,\left[h_{i} T_{t r p}^{\Delta l, \Delta \theta}(i, \delta, \tau \oplus j, \tau)-\lambda_{(i, \tau \oplus j)}\right]\right)
\end{aligned}
$$

Note that by the uniformity assumption, the above argument does not depend on the value of $\tau$. Therefore, one only needs to calculate $\xi$ once for all $\tau$ values. Thus, the computational time for each $V(\delta, \tau)$ can be significantly reduced.

\section{B. Reducing the Number of Times $V(\delta, \tau)$ is Computed}

Intuitively, some form of symmetry can be expected for the optimally placement of TRPs. For example, if there are only three available TRPs, one can expect the three TRPs to be placed uniformly around the AP. To describe such symmetry for a larger number of TRPs, we note that if one divides the network into a number of equal-size sectors, one may expect that the optimal TRP positions in each sector have the same pattern. In the following theorem, we state that the Lagrangian relaxation iterative algorithm can perform properly if one assumes a certain level of symmetry in the optimal placement of TRPs.

Theorem 2: If the network is divided into $m$ equal size sectors, where $N_{\text {sec }}=\frac{N}{m} \in \mathbf{Z}^{+}$and $\Delta_{s e c}=\frac{\theta_{\max }}{m} \in \mathbf{Z}^{+}$, and all sectors have the same set of Lagrange multipliers at the $n$-th iteration, i.e., $\lambda_{(i, j)}^{n}=\lambda_{\left(i, j \oplus k \Delta_{s e c}\right)}^{n}$, where $1 \leq k<m$ and $n \geq 1$, the same set of $N_{\text {sec }}$ TRPs will be allocated to each sector at the $n$-th iteration, i.e., $X_{(\delta, \tau)}^{n}=X_{\left(\delta, \tau \oplus k \Delta_{s e c}\right)}^{n}$. Consequently, all sectors have the same set of Lagrange multipliers at the $(n+1)$-th iteration, i.e., $\lambda_{(i, j)}^{n+1}=\lambda_{\left(i, j \oplus k \Delta_{s e c}\right)}^{n+1}$.

The proof of this theorem is given in Appendix II. By dividing the network into a number of equal-size sectors, we only need to rank the TRP candidate sites in one sector, and the other sectors will select the same set of TRP candidate sites by symmetry, and will update their Lagrange multipliers to the same set of values. Recall that, in the iterative algorithm in Section IV-C, the purpose of ranking the TRP candidate sites is to find the optimal values of $\mathbf{X}$ and $\mathbf{Y}$ in Step 2 and in turn find the subgradient for the current iteration. By using Theorem 2, we can start from the minimum sector size (i.e., $m=N$ ) and 
gradually increase the sector size while calculating the correct subgradient values.

Given $N$ TRPs to be placed into the network, we first perform a prime number decomposition for the number $N$ and save it in ascending order in vector $W$. It is clear that the number of ways that we can sectorize the network, denoted by the variable num_way, equals the length of $W$. For example, if $N=8$, then $W=\left[\begin{array}{llll}1 & 2 & 2 & 2\end{array}\right]$. Hence, we can sectorize the network into $8,4,2$, or 1 equal-size sectors, i.e., num_way $=4$. Then, by Theorem 2, in Section IV-C we need to consider only one sector (at any level of sectorization), instead of the entire network, as long as only one subgradient (i.e., only one sector-wide optimal solution) is found in Step 2 of the iterative algorithm. When more than one subgradient is found, we combine neighboring sectors to form the next coarser level of network sectorization, to allow the algorithm to select different subgradients in the subdifferential that corresponds to the combination of the multiple optimal solutions. ${ }^{8}$

The following pseudo code explains how the iterative algorithm in Section IV-C can be amended by the above procedure to significantly reduce its computational complexity:

- Step 0: At initiation, define the following variables:

- state $=1$, which represents the current state of the algorithm.

- current_num_sector $=N$, which represents the number of equal-size sector in the current iteration of the algorithm.

- current_num_TRP_per_sector $=1$, which represents the number of TRP to be allocated in each sector at the current iteration.

- Step 1: Step 1 of Section IV-C

- Step 2a: Step 2 of Section IV-C

- Step 2b: If the current_num_TRP_per_sector+1 smallest values of $V_{(\delta, \tau)}$ are equal and state $<$ num_way, do the following:

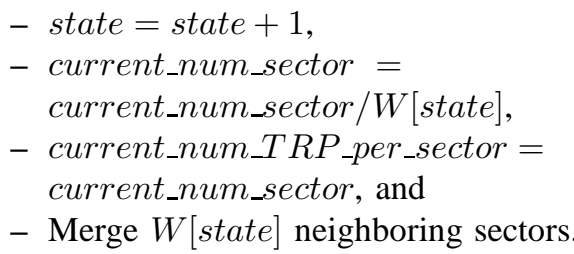

- Step 3: Step 3 of Section IV-C

- Step 4: Step 4 of Section IV-C

- Step 5: Step 5 of Section IV-C

\section{AN IMPLEMENTATION EXAMPLE}

In this Section, as a case study, we first describe an example implementation of the TRP mechanism for WLAN capacity improvement. We then study the optimal TRP placement in such a system, following an IEEE 802.11g-like bit rate model with large scale path-loss and Rayleigh fading.

\footnotetext{
${ }^{8}$ In this work, when multiple optimal solutions are found at any iteration, we choose one at random.
}

\section{A. Relaying and Rate Adaptation Mechanism}

The relaying and rate adaptation algorithm described here follows closely [15] in its operation principle. As explained in Section III, the proposed optimization framework is applicable as long as the TRP mechanism follows certain properties regarding relaying and rate adaptation. Thus, the implementation details presented in this section serve only as an example and are not mandatory.

We may employ a simple link statistics collection mechanism, following existing works which use receiver-initiated channel condition measurements [25][26]. For example, all nodes may listen to ongoing data and control packets. By extracting the address of the sender from the packet header and by measuring the signal strength of a packet, a node can record the channel condition between itself and another node. Moreover, all nodes periodically report to the AP the channel measurements that they recorded. Thus, the AP has up-to-date channel conditions, which is used to decide which TRP a MH should use. The AP will send a packet to a $\mathrm{MH}$ if the $\mathrm{MH}$ need to change the TRP that it is assigned.

For data packet relaying, a triangular handshake approach may be used. In the standard IEEE 802.11 DCF protocol, the RTS/CTS handshake is used for each unicast packet transmission to prevent collision. In [25][26], this handshake is further utilized to probe the channel condition. Following these principles and considering backward compatibility, we may follow a relaying approach similar to $r$ DCF which was first proposed in [15]. Fig. 6 illustrates how the triangular handshake is performed. If the source has a packet to send to the destination, and the TRP is assigned to this transmission, the source sends a new packet, called relay RTS (RRTS1) to the TRP. By sensing the signal strength of the RRTS1, the TRP determines the transmission rate, denoted $R_{1}$, that can be achieved from the source to itself. Upon receiving RRTS1, the TRP generates another relay RTS (RRTS2) and sends it to the destination after a SIFS. $R_{1}$ is piggyback in the RRTS2 packet. Again, by sensing the signal strength of RRTS2, the destination can determine the achievable transmission rate between the TRP and itself, denoted $R_{2}$. When a transmission via the TRP is possible, the destination will send a relay CTS (RCTS), which piggybacks $R_{1}$ and $R_{2}$, to the source. Once the source receives the RCTS, it sends the data packet to the TRP with rate $R_{1}$, and the TRP retransmits the data packet to the destination with rate $R_{2}$ after SIFS. If the packet is correctly received by the destination, it replies and ACK to the source. If transmission is not possible or fails, the sender can detect it with a timeout mechanism similar to the standard DCF. ${ }^{9}$

\section{B. IEEE 802.11g Bit Rate Model and Packet Transmission Time}

In this subsection, we derive the expected packet transmission time based on the IEEE $802.11 \mathrm{~g}$ bit rate model, which

\footnotetext{
${ }^{9}$ When the source directly communicates with the destination, the source sends a RTS to the destination and the destination senses the signal strength of this RTS and reply the appropriate rate to the source via the CTS package [25].
} 


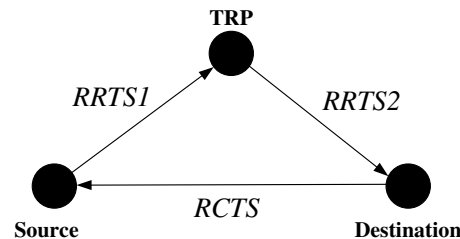

Fig. 6. An illustration of the triangular handshake.

will be used in our numerical analysis in Section VII. Suppose there are $M$ data rates, denoted $r_{1}, r_{2}, \ldots, r_{M}$, supported by the physical layer. Reliable communication by using rate $r_{m}$ can be realized only if the signal strength at the receiver is above a certain threshold, say $\eta_{m}$. Consequently, for the set of $M$ data rates, there is a set of $M$ thresholds, $\eta_{1}, \ldots, \eta_{M}$. We further define $\eta_{0}=0$ and $\eta_{M+1}=\infty$. For example, in IEEE 802.11g [30], there are 11 different bit rates, and the minimal threshold for each bit rate is specified by the standard.

We study the case where the following large-scale propagation model is applicable [31]:

$$
P_{r}=\frac{P}{d^{\alpha}},
$$

where $P$ is the reference signal power measured at one meter away from the transmitter, $P_{r}$ is the average signal power measured at $d$ meters away from the transmitter, and $\alpha$ is a positive constant representing the path loss exponent. The reference power $P$ can be obtained via field measurement or calculated using the following free space path loss formula [31]:

$$
P=\frac{P_{t x} G_{t} G_{r} \lambda^{2}}{(4 \pi)^{2} L}
$$

where $P_{t x}$ is the transmitted power, $G_{t}$ is the transmitter antenna gain, $G_{r}$ is the receiver antenna gain, $\lambda$ is the wavelength of the transmitted signal, and $L \geq 1$ is a loss factor not related to propagation. In our numerical analysis, $L$ is set to 1 , and the other parameters are taken from the specifications for commercial APs and WLAN interface cards.

In addition to large scale propagation, multipath fading may have a prominent effect on reliable communication. Under Rayleigh fading, the instantaneous power, $\gamma$, is exponentially distributed with the probability density function

$$
p(\gamma)=\frac{1}{P_{r}} e^{\frac{-\gamma}{P_{r}}},
$$

where $P_{r}$ is the average power of $\gamma$. Consequently, the probability that a transmitter with reference power $P$ can transmit at rate $r_{m}$, to a receiver at distance $l$, where $l>1$, is

$$
p\left(r_{m}, l, P\right)=\int_{\eta_{m}}^{\eta_{m+1}} \frac{l^{\alpha}}{P} e^{\frac{-\gamma l^{\alpha}}{P}} d \gamma,
$$

where $m=1,2, \ldots, M$. Furthermore, in some instances, the receiver can be located in a deep-fade area, i.e., is experiencing bad channel condition. The probability of these instances, where the transmitter cannot transmit in any data rate, is

$$
p_{f}(l, P)=\int_{\eta_{0}}^{\eta_{1}} \frac{l^{\alpha}}{P} e^{\frac{-\gamma l^{\alpha}}{P}} d \gamma
$$

while the probability that the transmitter can transmit successfully is

$$
p_{s}(l, P)=1-p_{f}(l, P)=\sum_{m=1}^{M} p\left(r_{m}, l, P\right) .
$$

In the above triangular handshake algorithm, the RRTS1 and RRTS2 require probing time, denoted $T_{\text {probe }} \cdot{ }^{10}$ Hence, $T_{\text {probe }}$ is needed to test the channel and decide the transmission rate before the actual data transmission can take place. If probing determines that the channel condition does not allow a successful transmission, i.e., either one of the two links is unavailable, the source will give up its transmission opportunity, and probe the channel again later. The wasted channel probing time adds to the total packet transmission time.

Let $T_{g}\left(d_{1}, d_{2}, P_{1}, P_{2}, x\right)$ be a random variable that represents the packet transmission time of an $x$-bit packet, and let $S$ and $F$ be the events of "good" and "bad" channel states respectively. The expected value of this packet transmission time is derived in (28), where $\sigma$ is the time for an SIFS. Rearranging the terms in (28), we have the expected packet transmission time

$$
\begin{aligned}
& T\left(d_{1}, d_{2}, P_{1}, P_{2}, x\right) \\
= & E\left[T_{g}\left(d_{1}, d_{2}, P_{1}, P_{2}, x\right)\right] \\
= & \frac{T_{\text {probe }}}{p_{s}\left(d_{1}, P_{1}\right) p_{s}\left(d_{2}, P_{2}\right)}+ \\
& {\left[\sum_{m=1}^{M} \sum_{n=1}^{M} \frac{p\left(r_{m}, d_{1}, P_{1}\right) p\left(r_{n}, d_{2}, P_{2}\right)}{p_{s}\left(d_{1}, P_{1}\right) p_{s}\left(d_{2}, P_{2}\right)}\left[\frac{x}{r_{m}}+\frac{x}{r_{n}}+\sigma\right]\right] . }
\end{aligned}
$$

If no relay is used, the one-hop expected packet transmission time, $T(l, P, x)$, can be calculated in a similar fashion.

\section{NUMERICAL ANALYSIS}

In this section, we present numerical results from the proposed optimization methods and evaluate the capacity improvement from using tetherless relay points in an outdoor WLAN. Unless otherwise stated, the system parameters such as signal power thresholds, antenna gains, and transmitter powers are taken from the CISCO Aironet 1100 Series AP and mobile NIC specifications [32]. The other system parameters are selected based on a typical outdoor environment.

\section{A. Convergence of Lagrangian Iteration and Effectiveness of Simplifications}

In Fig. 7, we show the convergence of the simplified algorithm in two typical network scenarios with channel path loss exponent, $\alpha=2.2$ and $\alpha=2.6$. For both scenarios, the network provides a coverage area of 400 meters in radius, and 16 TRPs are available to be placed in this network. Both the $\mathrm{AP}$ and TRP are equipped with a $10 \mathrm{dBm}$ transmitter, while the mobile hosts use a $5 \mathrm{dBm}$ transmitter. All transmitters and receivers have $2.2 \mathrm{dBi}$ antenna gain. The network occupies

\footnotetext{
${ }^{10}$ Since CTS and ACK require constant amount of time for every transmission with or without using a TRP, they are ignored in our packet transmission time calculation.
} 


$$
\begin{aligned}
& E\left[T_{g}\left(d_{1}, d_{2}, P_{1}, P_{2}, x\right)\right] \\
& =E\left[T_{g}\left(d_{1}, d_{2}, P_{1}, P_{2}, x\right) \mid F\right]\left(1-p_{s}\left(d_{1}, P_{1}\right) p_{s}\left(d_{2}, P_{2}\right)\right)+E\left[T_{g}\left(d_{1}, d_{2}, P_{1}, P_{2}, x\right) \mid S\right] p_{s}\left(d_{1}, P_{1}\right) p_{s}\left(d_{2}, P_{2}\right) \\
& =\left[E\left[T_{g}\left(d_{1}, d_{2}, P_{1}, P_{2}, x\right)\right]+T_{\text {probe }}\right]\left(1-p_{s}\left(d_{1}, P_{1}\right) p_{s}\left(d_{2}, P_{2}\right)\right)+ \\
& \\
& \quad\left[\sum_{m=1}^{M} \sum_{n=1}^{M} \frac{p\left(r_{m}, d_{1}, P_{1}\right) p\left(r_{n}, d_{2}, P_{2}\right)}{p_{s}\left(d_{1}, P_{1}\right) p_{s}\left(d_{2}, P_{2}\right)}\left[\frac{x}{r_{m}}+\frac{x}{r_{n}}+\sigma+T_{\text {probe }}\right]\right] \times p_{s}\left(d_{1}, P_{1}\right) p_{s}\left(d_{2}, P_{2}\right)
\end{aligned}
$$

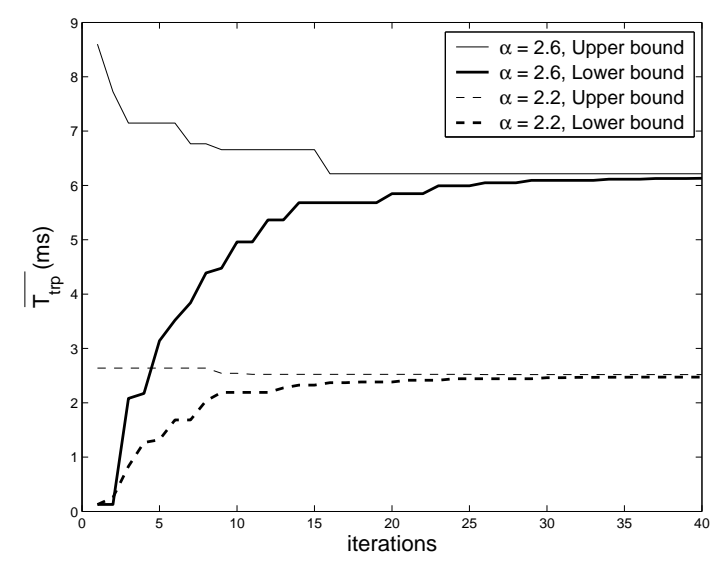

Fig. 7. Example convergence of the Lagrangian relaxation iterative algorithm.

a $20 \mathrm{MHz}$ channel in the $2.4 \mathrm{GHz}$ spectrum. The combined length of an uplink and a downlink packet is set to $2 \mathrm{k}$ bytes, and $70 \%$ of downlink traffic is assumed. By default, the network is discretized into 100 thousand cells, corresponding to approximately 5 square meters per cell on average.

As shown in Fig. 7, for both scenarios, the difference between the upper bound and the lower bound converges to less than $2 \%$ of the lower bound value in less than 40 iterations. In fact, for all system parameters that we have tested, the difference between the upper bound and the lower bound always converges to less than $2 \%$ of the lower bound value within 200 iterations. Similar results are omitted for brevity.

The proposed simplification schemes also significantly reduce the run time per iteration. To demonstrate this improvement, the same set of network parameters have been tested by using the algorithm with and without the proposed simplifications on the same computer. Furthermore, because of the large convergence time of the algorithm without the proposed simplifications, the network is discretized into 10 thousand cells instead of 100 thousand cells.

Let $L B(t)$ and $U B(t)$ be the lower bound and upper bound calculated by the algorithm at time $t$ respectively. We define optimality gap as

$$
O p t \_g a p(t)=100 \times \frac{U B(t)-L B(t)}{L B(t)} .
$$

As shown in Fig.8, by using the proposed simplifications, the optimality gap drops below $2 \%$ in less than 5 minutes, while it takes more than an hour to achieve the same results without using the simplifications. Note that 10 thousand cells correspond to 50 square meters per cell on average.

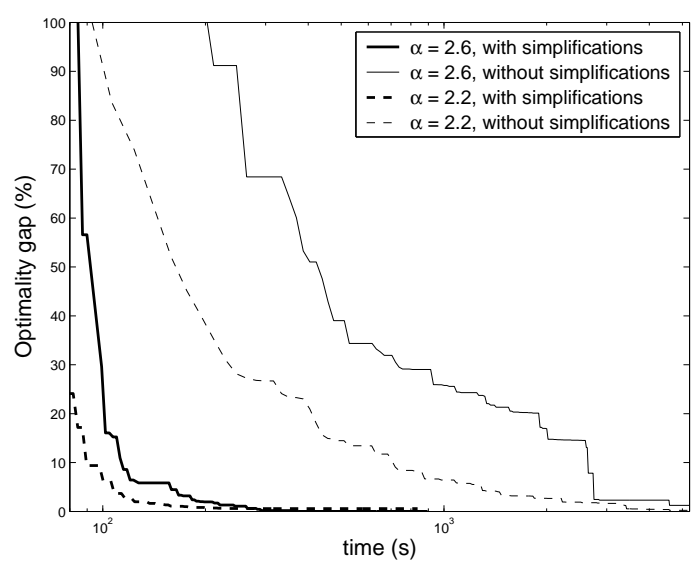

Fig. 8. Run time improvement with the proposed simplifications in typical network scenarios.

To achieve more practical, finer granularity, exponentially more computation will be necessary due to the NP-hardness of the TRP placement problem. In this case, the proposed simplifications can significantly reduce the run time of the Lagrangian relaxation iterative algorithm.

\section{B. Effect of System Parameters on TRP Placement and Per- formance Gain}

In this subsection, we discuss the benefit of the strategically placed TRPs with respect to different system parameters. We are interested in four system parameters: path loss exponent $(\alpha)$, proportion of downlink data $(\beta)$, power of the AP and TRP over power of MH ratio $\left(P_{a t} / P_{m}\right)^{11}$, and the number of TRPs $(N)$. For each set of parameters, our analysis and optimization procedure produce an optimal placement of TRPs. Example of such placements are shown in Fig. 9.

We study two throughput capacities of the network, $C_{a p}$ and $C_{t r p}$, without TRPs and with optimally placed TRPs, respectively as defined in (2). Thus, $C_{a p}$ and $C_{t r p}$ are defined as follows:

$$
C_{a p}=\frac{x}{\overline{T_{a p}}} \quad, \quad C_{t r p}=\frac{x}{\overline{T_{t r p}\left(\underline{\delta^{*}}, \underline{\tau^{*}}\right)}},
$$

where $\left(\underline{\delta^{*}}, \underline{\tau^{*}}\right)$ represents the optimal TRP location(s) computed by the Lagrangian relaxation iterative algorithm. We define the performance gain of utilizing the TRPs as

$$
\text { Gain }=100 \times \frac{C_{t r p}-C_{a p}}{C_{a p}} .
$$

\footnotetext{
${ }^{11}$ In this study, we assume the AP and TRP have the same reference power, which is denoted as $P_{a t}$.
} 


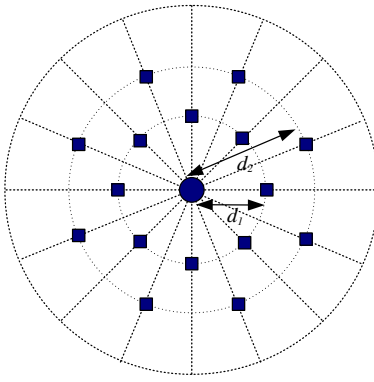

a. 16-TRP two-tier configuration

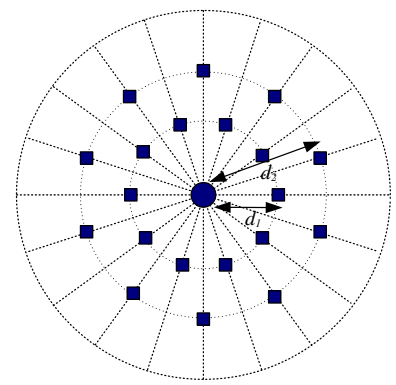

b. 20-TRP two-tier configuration
Parameters: coverage radius $=400 \mathrm{~m}, x=2 \mathrm{k}$ bytes, $\lambda=0.125 \mathrm{~m}, G_{t}=G_{r}=2.2 \mathrm{dBi}$, AP's and TRP's $P_{t x}=10 \mathrm{dBm}$, MH's $P_{t x}=5 \mathrm{dBm}$,

1. $\alpha=2.4,2.6,2.8$ and $\beta=0.7$,

2. $\alpha=2.6$ and $\beta=0,0.25,0.5,0.75$

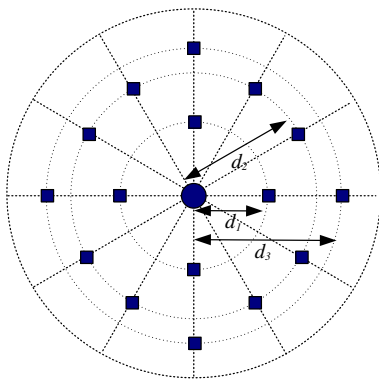

c. 16-TRP three-tier configuration

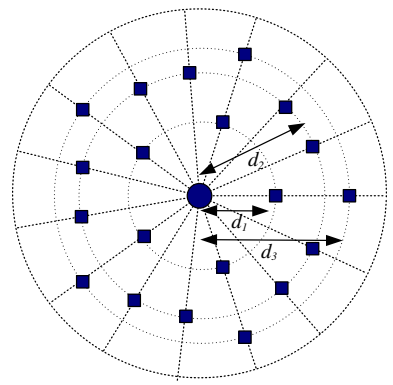

d. 20-TRP three-tier configuration
Parameters: coverage radius $=400 \mathrm{~m}, x=2 \mathrm{k}$ bytes, $\lambda=0.125 \mathrm{~m}, G_{t}=G_{r}=2.2 \mathrm{dBi}$, AP's and TRP's $P_{t x}=15 \mathrm{dBm}, \alpha=2.4, \beta=0.7$, 1. $P_{a t} / P_{m}=10 \mathrm{~dB}, 15 \mathrm{~dB}, 20 \mathrm{~dB}$.

Fig. 9. Different TRP placement configurations with different number of TRPs with respect to different system parameters.

In the following, the relationship among the four system parameters with respect to the optimal TRP placement and performance gain will be discussed.

Moreover, we also compare the optimal TRP performance gain with results from random placement of the same number of TRPs. In the random placement scheme, for each set of system parameters, 100 different random TRP placements are generated, and we report the average performance gain of relaying using these TRPs.

1) Path loss Exponent: We study the effect of the path loss exponent, $\alpha$, and the number of TRPs, $N$, in Fig. 10 and Fig. 11. All other parameters are set as in Section VII-A.

Fig. 10 and Fig. 11 show the performance gains and optimal TRP placements with respect to different path loss exponents respectively. When there are 4,8 or 12 TRPs, the solution calculated by the algorithm converges to a singletier configuration, where the TRPs are uniformly distributed around and with a displacement $d_{1}$ meters away from the AP. When there are 16 or 20 TRPs available, the algorithm converges to a two-tier configuration, which are shown in Fig. 9a, and Fig. 9b.

As we can see from Fig. 11, when the path loss exponent is small, the TRPs are located further away from the AP. This can be explain by the fact that when the path loss exponent is low, the MHs which are located close to the AP can already transmit at very high rates; thus, only a small number of MHs which are located very far away from the AP can benefit from the TRPs. Moreover, since the signal will not

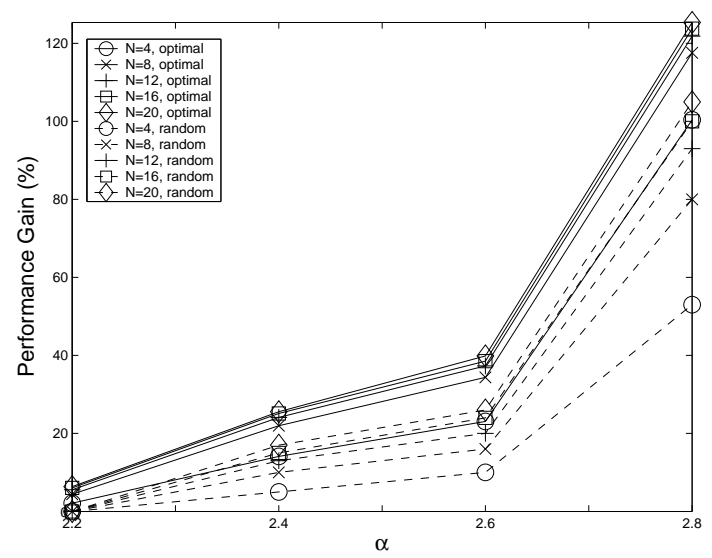

Fig. 10. Performance gain with respect to different path loss exponent and number of TRPs.

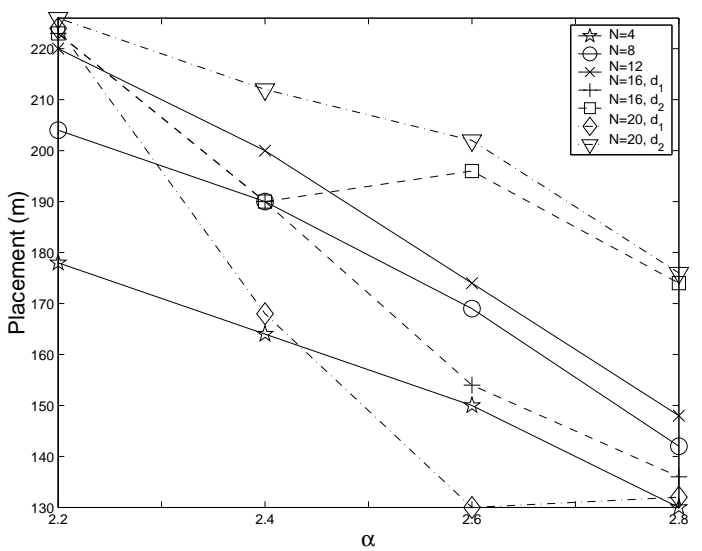

Fig. 11. Optimal placement of TRPs with respect to different path loss exponents and number of TRPs.

attenuate severely in these scenarios, as shown in Fig. 10, the performance gains are very low. However, when the path loss exponent is large, the performance gain of using TRPs can be higher than $120 \%$. The path loss exponent determines how fast the signal decays when it travels through a distance. Therefore, as the path loss exponent increases, the beneficial effects of the TRPs become more significant. When the path loss exponent is large, the TRPs are located closer to the AP. This implies that the distanced MHs receive less benefit from the TRPs when the path loss exponent is large. This result suggests that when the path loss exponent is large, it is more beneficial to allocate more TRPs to help MHs that are relatively closer to the AP. In other words, in high channel attenuation environments, the marginal benefits provided by the TRPs to the distanced MHs are less than that to the MHs which are near the AP.

In all cases, the effect of diminishing return is observed as the number of TRPs increases. The marginal benefit of an addition of 4 TRPs is only a few percent for $N \geq 8$. This observation suggests that when the difference of transmitter's power between the MH and AP/TRP is not high, and downlink traffic dominates uplink traffic, a small number of TRPs is sufficient to achieve reasonable performance gain.

Furthermore, we note that the optimally placed TRPs provide significant performance advantage over the average case 


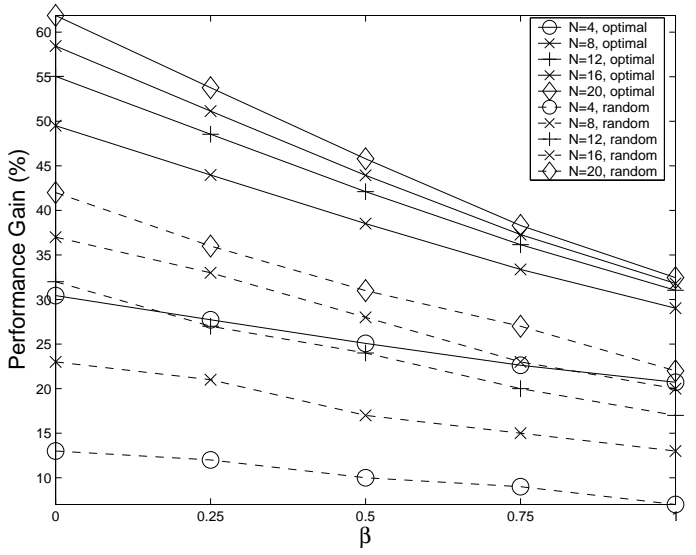

Fig. 12. Performance gain with respect to proportion of downlink data and the number of TRPs.

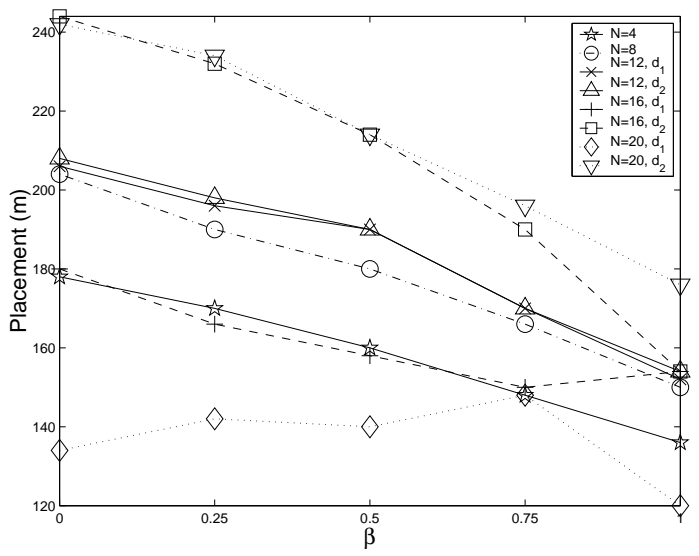

Fig. 13. Optimal placement of TRPs with respect to different proportion of downlink data and number of TRPs.

of random TRP placement, especially when the numbers of TRPs are moderate to small. The same observation can be made in Figures 12 and 14 below.

2) Proportion of Downlink and Uplink Traffic: We study the effect of the proportion of downlink data $(\beta)$ and the number of TRPs $(N)$ on an outdoor network in Fig. 12 and Fig. 13. The system parameters are the same as in Section VIIA, except the path loss exponent is set to 2.6 , and the downlink and uplink packet lengths are $2 \beta \mathrm{k}$ bytes and $2(1-\beta) \mathrm{k}$ bytes respectively.

From Fig. 12, the performance gain increases as the proportion of uplink data, $(1-\beta)$, increases. This is, again, because the MH's transmitter has less power compared with that of the AP and TRPs. As the amount of data needed to be transmitted by the MH's transmitter increases, the beneficial effect of the TRPs becomes more and more significant; this in turn results in higher performance gain.

When there are 4 or 8 TRPs, the algorithm converges to a single-tier configuration regardless of the proportion of downlink data. When there are 12 TRPs, the algorithm converges to a two tier configuration only when the uplink traffic dominates the downlink traffic. When the number of TRPs is large, 16 or 20 TRPs, the algorithm always converges to a two-tier configuration, which are shown in Fig. 9a, and

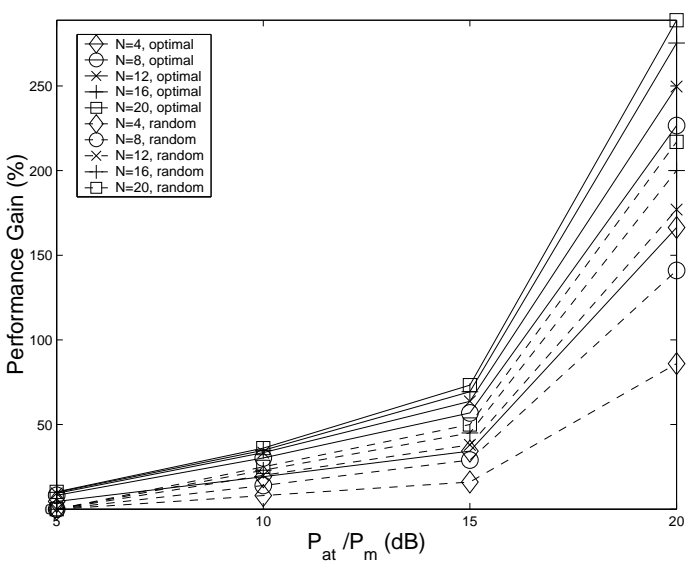

Fig. 14. Performance gain with respect to different $P_{a t} / P_{m}$ and number of TRPs.

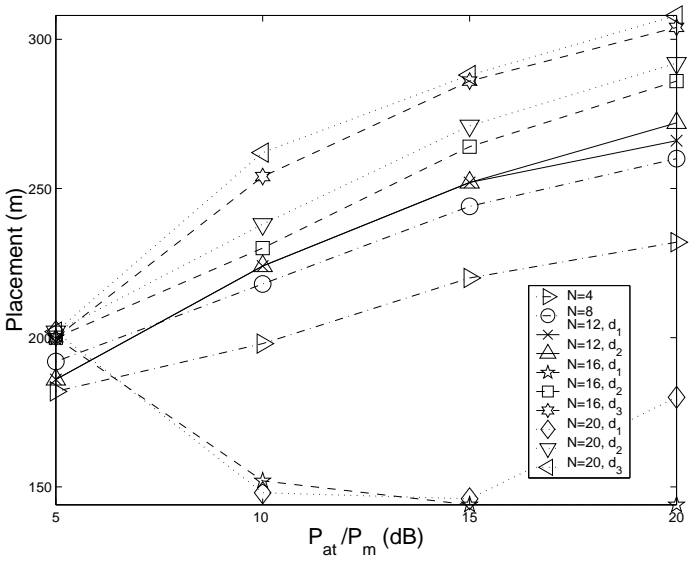

Fig. 15. Optimal placement of TRPs with respect to different $P_{a t} / P_{m}$ and number of TRP.

Fig. 9b. From Fig. 13, the strategically placed TRPs are more spread out and further away from the AP when the proportion of downlink data, $\beta$, is low. This is because the MHs have less transmit power compared with that of AP and TRP. When the proportion of downlink data is low, the MHs have more to transmit. When MHs have more to transmit, MHs that are located far away from the AP need more help compared with those located close to the AP. Therefore, in order to minimize the expected packet transaction time, the TRPs should be placed further away from the AP.

Again, in all cases, the effect of diminishing return is observed. This effect is less severe when there is only uplink traffic, when the difference in performance gain between 8 TRPs and 20 TRPs is only $12 \%$. In the next subsection, we describe scenarios where using a larger number of TRPs can result in more significant return.

3) AP/TRP to MH Power Ratio: We study the effect of the AP, TRP to MH transmitter power ratio $\left(P_{a t} / P_{m}\right)$ and the number of TRPs on an outdoor network in Fig. 14 and Fig. 15. The system parameters are the same as in Section VII-A, except the path loss exponent is set to 2.4 , and the AP's and TRP's transmitter power are $15 \mathrm{dBm}$, while the MH's transmitter power varies depend on the $P_{a t} / P_{m}$ ratio.

From Fig. 14, the performance gain increases as the $\frac{P_{a t}}{P_{m}}$ 
ratio increases. The $\frac{P_{a t}}{P_{m}}$ ratio represents the difference of transmitter power between AP/TRP and mobile hosts. When MH's transmitter power is low, the effect of the TRPs becomes more significant. Hence, the performance gain is high when the mobile hosts have less powerful transmitters. Moreover, when the $\frac{P_{a t}}{P_{m}}$ ratio is high, the effect of diminishing return on larger number of TRP is relatively low. Thus, with high power difference between the AP/TRP and MHs, the marginal benefit of additional TRP is relatively high. As shown in Fig. 14, the difference in performance gain between using 4 and 20 TRPs can be greater than $100 \%$ when the power difference is large. In other words, the installation of a larger number of TRPs in such network can be justified.

When there are 4 or 8 TRPs, the algorithm always converges to a single-tier configuration regardless of the power difference. In the case of 12 TRPs, the algorithm converges to a single-tier configuration when the power difference is small and to a two-tier configuration when the power difference is large. When there are 16 and 20 TRPs, the results produced by the Lagrangian algorithm are more interesting; the configurations are shown in Fig. 9c, and Fig. 9d.

From Fig. 15, the optimally placed TRPs are more spread out when the $\frac{P_{a t}}{P_{m}}$ ratio is high. Moreover, more TRPs are located closer to the edge of the network. This is because the MHs can benefit more from the TRPs when the MH's transmitter power is relatively low. Therefore, in order to minimize the network expected packet transaction time, more TRPs are located closer to the edge of the network so that the distanced MHs can receive more help.

\section{CONCLUSIONS}

In this work, we investigate the strategic placement of TRPs to enhance the throughput capacity of a WLAN in environments where wiring is practically or economically infeasible. We have presented a relaying architecture which increases the communication rate between a $\mathrm{MH}$ and the access point, developed an analytical model for performance evaluation and TRP placement optimization, and proposed a Lagrangian relaxation iterative algorithm to solve a discrete version of the TRP placement optimization problem. The proposed framework can be generalized to fit different channel models, network configurations, environmental constraints and user behaviors. In particular, we have investigated the TRP placement problem in a WLAN with IEEE 802.11g-like bit rates under Rayleigh fading, with a sample relaying and rate adaptation algorithm. Moreover, simplifications tailored for this problem have been proposed to significantly reduce the computational complexity of the proposed iterative algorithm.

Using the proposed numerical analysis framework, we have showed that in most cases, by using strategically placed TRPs, the network capacity can be significantly improved. Moreover, for different network environments, we quantify the effects of the AP/TRP power to MH power ratio, network path loss exponent, proportion of downlink data, and number of available TRPs, with respect to the optimal TRP placement and its resulting throughput capacity improvement. Finally, we note that, given a set of network parameters, the proposed algorithm and performance results can be used by network designers to compute the optimal placement of TRPs and provide design guidelines to justify the tradeoff between additional hardware cost and system performance gain.

\section{APPENDIX I \\ PROOF OF THEOREM 1}

Proof: Because of symmetry, we only consider the upper half of the sector, i.e., $0<j<\frac{\theta_{\max }}{2}$. Given $i$, $\delta$, and $\tau$, clearly $T_{t r p}^{\Delta l, \Delta \theta}(i, \delta, j \oplus \tau, \tau)$ increases as $j$ increases, since as the angle between the TRP and the MH with respect to the AP increases, the distance between the TRP and the MH increases. Moreover, $T_{a p}^{\Delta l}(i)$ does not depend on $j$. Hence there exists a minimal $\xi(i, \delta)$ such that for all $\frac{\theta_{\max }}{2}>j>\xi(i, \delta)$, $T_{t r p}^{\Delta l, \Delta \theta}(i, \delta, j \oplus \tau, \tau)>T_{a p}^{\Delta l}(i) . \xi$ is simply the maximum of all $\xi(i, \delta)$, i.e., $\xi=\max _{i, \delta} \xi(i, \delta)$. The same argument applies to the lower half of the network.

\section{APPENDIX II \\ PROOF OF THEOREM 2}

Proof: First, we show that if each sector has the same set of Lagrange multipliers, the same set of TRP candidate sites will be selected at each sector, i.e., if $\lambda_{(i, j)}=\lambda_{\left(i, j \oplus k \Delta_{s e c}\right)}$, then $V_{(\delta, \tau)}=V_{\left(\delta, \tau \oplus k \Delta_{s e c}\right)}$, where $1 \leq k<m$. Since $T_{\text {trp }}^{\Delta l, \Delta \theta}(i, \delta, \tau \oplus j, \tau)=T_{t r p}^{\Delta l, \Delta \theta}\left(i, \delta, \tau \oplus j \oplus k \Delta_{s e c}, \tau \oplus k \Delta_{s e c}\right)$, we have

$$
\begin{aligned}
V_{(\delta, \tau)} & =\sum_{i=1}^{l_{\max }} \sum_{j=-\xi}^{\xi} \min \left(0,\left[h_{i} T_{t r p}^{\Delta l, \Delta \theta}(i, \delta, \tau \oplus j, \tau)-\lambda_{(i, \tau \oplus j)}\right]\right) \\
& =\sum_{i=1}^{l_{\max }} \sum_{j=-\xi}^{\xi} \min \left(0,\left[h _ { i } T _ { t r p } ^ { \Delta l , \Delta \theta } \left(i, \delta, \tau \oplus j \oplus k \Delta_{s e c}, \tau \oplus\right.\right.\right. \\
& =V_{\left(\delta, \tau \oplus k \Delta_{s e c}\right) .}
\end{aligned}
$$

Consequently, if a minimum $V_{(\delta, \tau)}$ is found in one sector, another minimal TRP candidate site will be found in another sector, $V_{\left(\delta, \tau \oplus k \Delta_{s e c}\right)}$, where $1 \leq k<m$. As a result, $N_{\text {sec }}$ TRP candidate sites will be selected at each sector. Furthermore, this implies $X_{(\delta, \tau)}^{n}=X_{\left(\delta, \tau \oplus k \Delta_{s e c}\right)}^{n}$.

Second, we show that if at the $n$-th iteration all sectors have the same set of Lagrange multipliers, then at the $n+1$-th iteration all sectors have the same set of Lagrange multipliers, i.e., if $\lambda_{(i, j)}^{n}=\lambda_{\left(i, j \oplus k \Delta_{s e c}\right)}^{n}$, then $\lambda_{(i, j)}^{n+1}=\lambda_{\left(i, j \oplus k \Delta_{s e c}\right)}^{n+1}$, where $1 \leq k<m$.

To show this is true, we first note that $Y_{(i, j),(0,0)}^{n}=1 \Longleftrightarrow$ $h_{i} T_{a p}^{\Delta l}(i)-\lambda_{(i, j)}^{n}<0$. Since $h_{i} T_{a p}^{\Delta l}(i)-\lambda_{(i, j)}^{n}=h_{i} T_{a p}^{\Delta l}(i)-$ $\lambda_{\left(i, j \oplus k \Delta_{\text {sec }}\right)}^{n}$, we have

$$
Y_{(i, j),(0,0)}^{n}=Y_{\left(i, j \oplus k \Delta_{s e c}\right),(0,0)}^{n} .
$$

Furthermore, we have $Y_{(i, j)(\delta, \tau)}^{n}=1 \Leftrightarrow X_{(\delta, \tau)}^{n}=$ 1 and $h_{i} T_{t r p}^{\Delta l, \Delta \theta}(i, \delta, j, \tau)-\lambda_{(i, j)}^{n}<0$. As shown before, $X_{(\delta, \tau)}^{n}=1 \Leftrightarrow X_{\left(\delta, \tau \oplus k \Delta_{s e c}\right)}^{n}=1$. Moreover, $\quad h_{i} T_{t r p}^{\Delta l, \Delta \theta}(i, \delta, j, \tau)-\lambda_{(i, j)}^{n}<0 \Leftrightarrow$ $h_{i} T_{t r p}^{\Delta l, \Delta \theta}\left(i, \delta, j \oplus k \Delta_{s e c}, \tau \oplus k \Delta_{s e c}\right)-\lambda_{\left(i, j \oplus k \Delta_{s e c}\right)}^{n}<$ 
0. Therefore $\quad \sum_{\delta=1}^{l_{\max }} \sum_{\tau=0}^{\theta_{\max }-1} Y_{(i, j),(\delta, \tau)}^{n}=$ $\sum_{\delta=1}^{l_{\max }} \sum_{\tau=0}^{\theta_{\max }-1} Y_{\left(i, j \oplus k \Delta_{\text {sec }}\right),\left(\delta, \tau \oplus k \Delta_{\text {sec }}\right)}^{n}$ Rearranging the index $\tau$, we have

$\sum_{\delta=1}^{l_{\max }} \sum_{\tau=0}^{\theta_{\max }-1} Y_{(i, j),(\delta, \tau)}^{n}=\sum_{\delta=1}^{l_{\max }} \sum_{\tau=0}^{\theta_{\max }-1} Y_{\left(i, j \oplus k \Delta_{s e c}\right),(\delta, \tau)}^{n}$.

By using (34) and (35) and putting them in (20), we have $\lambda_{(i, j)}^{n+1}=\lambda_{\left(i, j \oplus k \Delta_{s e c}\right)}^{n+1}$.

\section{REFERENCES}

[1] Y. Bejerano and R. Bhatia, "MiFi: A framework for fairness and QoS assurance in current IEEE 802.11 networks with multiple access points," in Proc. of IEEE INFOCOM, Hong Kong, China, March 2004, vol. 2, pp. $1229-1240$.

[2] S.Vasudevan, K.Papagiannaki, C.Diot, J. Kurose, and D. Towsley, "Facilitating access point selection in IEEE 802.11 wireless networks," in Proc. of ACM SIGCOMM/USENIX Internet Measurement Conference (IMC), Oct. 2005

[3] Y. Bejerano, S. Han, and L. Li, "Fairness and load balancing in wireless LANs using association control," in Proc. of ACM MOBICOM, Philadelphia, PA, U.S.A., Sept. 2004, pp. 315 - 329.

[4] P. Zerfos, G. Zhong, and S. Lu, "A software support infrastructure for wireless access routers," IEEE Journal on Selected Areas in Communications, vol. 23, no. 6, pp. 1253 - 1266, June 2005.

[5] O. Sharon and E. Altman, "On efficient polling MAC for wireless LANs," IEEE Transactions on Networking, vol. 9, no. 4, pp. 439 - 451, Aug. 2001.

[6] D. Qiao, S. Choi, A. Soomro, and K. G. Shin, "Energy-efficient PCF operation of IEEE 802.11a wireless LAN," in Proc. of IEEE INFOCOM, New York City, NY, U.S.A., June 2002, vol. 2, pp. 580 - 589.

[7] T. Kawata, S. Shin, A. Forte, and H. Schulzrinne, "Using dynamic PCF to improve the capacity for VoIP traffic in IEEE 802.11 networks," in Proc. of IEEE WCNC, New Orleans, LA, U.S.A., March 2005, vol. 3, pp. $1589-1595$.

[8] S. Kim, B. Kim, and Y. Fang, "Downlink and uplink resource allocation in IEEE 802.11 wireless LANs," IEEE Transactions on Vehecular Technology, vol. 54, no. 1, pp. 320-327, Jan. 2005.

[9] H. Chang and V. Misra, "802.11 link interference: A simple model and a performance enhancement," in Proc. of IFIP Networking, Waterloo, ON, Canada, May 2005.

[10] A. Vasan, R. Ramjee, and T. Woo, "ECHOS - enhanced capacity 802.11 hotspots," in Proc. of IEEE INFOCOM, Miami, FL, U.S.A., March 2005.

[11] C. E. Perkins, Ed., Ad Hoc Networking, Addison-Wesley Longman, 2001.

[12] Z. Haas, J. Deng, B. Liang, P. Papadimitratos, and S. Sajama, Wiley Encyclopedia of Telecommunications, John Wiley \& Sons, 2002.

[13] Y. Lin and Y. Hsu, "Multihop cellular: A new architecture for wireless communications," in Proc. of IEEE INFOCOM, Tel-Aviv, Israel, March 2000 , vol. 3, pp. $1273-1282$.

[14] H. Zhu and G. Cao, "On improving the performance of IEEE 802.11 with relay-enabled PCF," ACM/Kluwer Mobile Networking and Applications (MONET), vol. 9, pp. 423-434, 2004.

[15] H. Zhu and G. Cao, "rDCF: A relay-enabled medium access control protocol for wireless ad hoc networks," in Proc. of IEEE INFOCOM, Miami, FL, U.S.A., March 2005, vol. 1, pp. 12-22.

[16] H. Wu, C. Qiao, S. De, and O. Tonguz, "Integrated cellular and ad hoc relaying systems: iCAR," IEEE Journal on Selected Areas in Communications, vol. 19, no. 10, pp. 2105-2215, October 2001.

[17] Y. Bejerano, "Efficient integration of multihop wireless and wired networks with QoS constraints," IEEE Transactions on Networking, vol. 12, no. 6, pp. 1064- 1078, Dec. 2004

[18] F. Fitzek, D. Angelini andG. Mazzini, and M. Zorzi, "Design and performance of an enhanced IEEE802.11 MAC protocol for ad hoc networks and coverage extension for wireless networks," IEEE Wireless Communications, vol. 10, no. 6, pp. 30 - 39, Dec. 2003.

[19] R. Karrer, A. Sabharwal, and E. Knightly, "Enabling large-scale wireless broadband: The case for TAPs" in Proc. of Hotnets, Cambridge, MA, U.S.A., Nov. 2003.

[20] J. Camp, J. Robinson, C. Steger, and E. Knightly, "Measurement driven deployment of a two-tier urban mesh access network" in Proc. of ACM MobiSys, Uppsala, Sweden, Jun. 2006.
[21] R. Draves, J. Padhye, and B. Zill, "Routing in multi-radio, multi-hop wireless mesh network," in Proc. of ACM MOBICOM, Philadelphia, PA, U.S.A., Sept. 2004, pp. $114-128$.

[22] R. Draves, J. Padhye, and B. Zill, "Comparison of routing metrics for multi-hop wireless networks," in Proc. of ACM SIGCOMM, Portland, OR, U.S.A., Sept. 2004.

[23] A. So and B. Liang, "An efficient algorithm for the optimal placement of wireless extension points in rectilineal wireless local area networks," in Proc. of The Second Int. Conf. on Quality of Service in Heterogeneous Wired/Wireless Networks (QShine), Orlando, FL, U.S.A., August 2005, pp. $101-109$.

[24] "IEEE standard for information technology, local and metropoliton networks - specific requirements, part 11: Wireless LAN medium access control (MAC) and physical layer (PHY) specifications," Tech. Rep., IEEE Computer Society, 1999.

[25] G. Holland, N. Vaidya, and P. Bahl, "A rate-adaptive MAC protocol for multi-hop wireless networks," in Proc. of ACM MOBICOM, Rome, Italy, July 2001 , pp. $236-251$.

[26] B. Sadeghi, V. Kanodia, A. Sabharwal, and E. Knightly, "Opportunistic media access for multirate ad hoc networks," in Proc. of ACM MOBICOM, Atlanta, GA, U.S.A., Sept. 2002, pp. 24-35.

[27] A. Papoulis and U. Pillai, Probability, Random Variables and Stochastic Processes, McGraw Hill, 4 edition, 2002.

[28] L. Snyder, "Facility location under uncertainty: A review," IIE Transactions (under revision), Feb. 2005.

[29] R. K. Martin, Large Scale Linear and Integer Programming, Kluwer Academic Publishers, 1 edition, 1999.

[30] "IEEE standard for information technology, local and metropoliton networks - specific requirements, part 11: Wireless LAN medium access control (MAC) and physical layer (PHY) specifications- Amendment 4: Further higher-speed physical layer extension in the $2.4 \mathrm{GHz}$ band," Tech. Rep., IEEE Computer Society, 2003.

[31] T. S. Rappaport, Wireless Communications: Principles and Practice, Prentice Hall, 2 edition, 2001.

[32] "CISCO Aironet 1100 series access point: Data sheet," Tech. Rep., CISCO Systems, 2003.

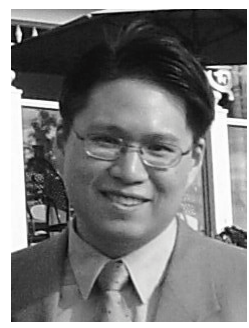

Aaron So (S'04) received B.Sc. (first-class honors) and M.Sc. degrees in Mathematics and Engineering from Queen's University in Kingston, Ontario, Canada in 2002 and 2003 respectively. He joined the Ph.D. program in the Department of Electrical and Computer Engineering at the University of Toronto in 2003. He received the Ontario Graduate Scholarship in 2003 and 2006, the Ontario Graduate Scholarship in Science and Technology in 2004 and 2005, and the Bell University Labs Scholarship in 2006 toward the completion of his Ph.D. dissertation. His $\mathrm{Ph} . \mathrm{D}$. research topic is strategic configurations of relay infrastructure in wireless networks.

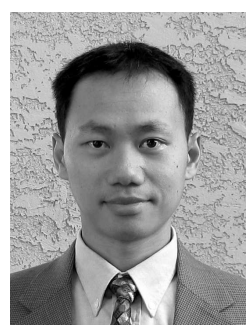

Ben Liang (S'94-M'01-SM'06) received honors simultaneous B.Sc. (valedictorian) and M.Sc. degrees in electrical engineering from Polytechnic University in Brooklyn, New York, in 1997 and the Ph.D. degree in electrical engineering with computer science minor from Cornell University in Ithaca, New York, in 2001. In the 2001 - 2002 academic year, he was a visiting lecturer and post-doctoral research associate at Cornell University. He joined the Department of Electrical and Computer Engineering at the University of Toronto as an Assistant Professor in 2002. His current research interests are in mobile networking and multimedia systems. He received an Intel Foundation Graduate Fellowship in 2000 toward the completion of his Ph.D. dissertation, the Best Paper Award at the IFIP Networking conference in 2005, and the Runner-up Best Paper Award at the International Conference on Quality of Service in Heterogeneous Wired/Wireless Networks in 2006. He is a senior member of IEEE and a member of ACM and Tau Beta Pi. He serves on the organizational and technical committees of a number of major conferences each year. 